\title{
Edith Stein: entre la psicología de William Stern y la fenomenología de Edmund Husserl
}

\section{Edith Stein between the psychology of William Stern and the phenomenology of Edmund Husserl}

\author{
Danilo Souza Ferreira \\ DOGTORADO EN HISTORIA \\ UNIVERSIDAD FEDERAL DE OURO PRETO- UFOP-MG / EDITH STEIN GIRGLE \\ danilosf1901@hotmail.com \\ ORGID 0000-0003-2548-5553
}

Recibido: 25/Julio/2020 - Aceptado: 30/Octubre/2020

http://dx.doi.org/10.7764/Steiniana.4.1.2020.4

Resumen: En este artículo traemos una breve biografia de Edith Stein. Tal trabajo se justifica por dos razones: la primera es la presentación de la filósofa, que es poco conocida por la academia latinoamericana, y la segunda razón es la relación íntima entre su vida y los acontecimientos históricos cruciales que marcaron la primera mitad del siglo XX, principalmente la historia de Alemania en el período moderno, así como la Primera Guerra Mundial, la República de Weimar y el surgimiento del nazismo en Alemania, además de la aparición de corrientes teóricas como la fenomenología. Buscaremos en particular, en este texto, describir las influencias que Stein ha recibido del psicólogo William Stern, miembro de la escuela y profesor de Wurzburg en Breslau, y del fenomenólogo Edmund Husserl, quien impartió clases de filosofía en Gotinga, y con quien desarrolló su reflexión sobre el papel de la empatía, como elemento constitutivo de la relación entre los individuos, así como su relación con la historia.

Palabras-chave: Edith Stein, fenomenología, historia intelectual, William Stern, Edmund Husserl.

Abstract: In this article we bring a brief biography of Edith Stein. Such work is justified for two reasons: the first is the presentation of the philosopher, who is little known by the Latin American academy, and the second reason is the intimate relationship between her life and the crucial historical events that marked the first half of the 20th century, mainly the History of Germany in the modern period, as well as the First World War, the Weimar Republic and the rise of 
Nazism in Germany, in addition to the emergence of theoretical currents such as phenomenology. We will seek in particular, in this text, to describe the strong influences that Stein suffered from the psychologist William Stern, a member of the Wurzburg school and professor in Breslau, and from the phenomenologist Edmund Husserl, who taught Philosophy classes in Gottingen, and with whom she developed her reflection on the role of empathy as constitutive of the relationship between individuals, as well as its relationship with history.

Keywords: Edith Stein, phenomenology, intellectual history, William Stern, Edmund Husserl.

\section{INTRODUCGIÓN}

Buscaremos en este artículo presentar la formación académica de la filósofa Edith Stein, especialmente durante el período en el que estudió en la Universidad de Breslau, donde asistió a las disciplinas Historia y Filología y posteriormente en la Universidad de Göttingen donde estudió la fenomenología de Edmund Husserl, para lograr este objetivo utilizamos el enfoque de la historia intelectual para analizar el trasfondo biográfico del la intelectual, utilizamos en particular la biografía Vida de una familia judía y otros escritos autobiográficos y las cartas recopiladas en la primera edición de las obras completas titulada Escritos filosóficos Etapa fenomenológica: 1915-1920 estas obras nos permiten comprender cómo Edith Stein se construye como intelectual.

Alasdair Macintyre aborda el enfoque de Edith Stein sobre la corriente fenomenológica ya en los años de su formación en la Universidad de Breslau, en Alemania, donde los temas elegidos por Stein (Historia y Filología, especialmente germánica) formaban parte de las llamadas ciencias del espíritu. Más tarde, Stein asistió a las disciplinas de Filosofía, impartidas por Richard Honigswald, y de Psicología Experimental, impartidas por Louis Wiliam Stern, cuando, a su debido tiempo, se le presentaron los escritos de Edmund Husserl ${ }^{141}$.

Richard Honigswald, de origen judío, enseñó en Breslau desde el año 1906, desarrollando, hasta 1915, trabajos sobre la teoría de las Matemáticas y las Teorías de la Cognición. Se dedicó, en particular, a la Historia de la Filosofía a través 
de la inspiración del neokantismo, así como a Alois Riehl, con quien mantuvo un fuerte diálogo. En los años 1909-1915, Honigswald publicó artículos sobre Teoría de la Ciencia, teniendo como foco principal la Teoría de las Matemáticas, que lo acerca a los trabajos de Ernst Cassirer y Bruno Bauch. Más tarde, había escrito sobre la Historia de la filosofía y también sobre cuestiones de perfil historiográfico. La originalidad del proyecto de Honigswald y la percepción de que las experiencias históricas se presentarían a través de "mónadas" permiten "la validez del pensamiento y la voluntad en el mundo", lo que se puede ver en sus obras Fundamentos de la educación, desde 1918 y Pensamiento psicológico, $1921^{142}$.

En el libro Historia de una familia judía, Stein describe su período como estudiante en la Universidad de Breslau (específicamente en el cuarto capítulo, titulado Estrenando em la libertad académica) ${ }^{143}$. El autor señala cuán sorprendente es el hecho de que Honigswald y Stern fueran maestros contratados, Privatdozent, un término utilizado para referirse a los maestros que fueron admitidos para enseñar después de someterse a un examen especial, buscando enfatizar que uno de los factores que obstaculizó la contratación de ellos fue el hecho de que eran de origen judío.

El trasfondo judío de Stern y Honigswald fue un inconveniente para su carrera académica. La cátedra de psicología en Breslau no era rentable y Honigswald todavía era un "maestro contratado" y lo seguiría siendo durante muchos años, y más tarde se le dio la cátedra de psicología cuando Stern aceptó un contrato en Hamburgo, y muy tarde recibió el presidente de filosofia (Munich), por todo lo que sufrió visiblemente ${ }^{144}$.

La denuncia señalada por Edith Stein se relaciona con el hecho de que los profesores de origen judío y que fueron contratados, o Privatdozent, tenían que pasar por un proceso de admisión diferente, que consistía en la evaluación de sus tesis doctorales. Además, su salario no fue pagado por la universidad, sino a través de un acuerdo entre los estudiantes, quienes a su vez pagaron los sueldos de los maestros para enseñar las materias.

Aparentemente, esta situación de antisemitismo sombrío, presente desde

142 PETER ULRICH MERZ BENZ, Richard Honigswald und Norbert Elias - Von der Geschichtsphilosophie zur Soziologie. (Boon. Ed: Springer,1997), 133.

143 EDITH STEIN, Escritos Filosóficos Etapa fenomenológica: 1915-1920. (Madrid: El Carmen, 2002$), 200$.

144 EDITH STEIN, Vida de uma família judia e outros escritos autobiográficos. (São Paulo. Ed: Paulus, 2018) ,298. 
ese período en las universidades alemanas, así como en la sociedad en general, también estuvo presente en otras situaciones. Un ejemplo de esto es el caso del sociólogo alemán de origen judío, Georg Simmel, quien obtuvo un nombramiento como profesor en la Universidad de Estrasburgo, que, como señaló Marquard, era una universidad fuera del eje más importante, como los de Berlín, Munich o Heidelberg, en la que Simmel intentó ingresar en 1908, sin obtener la silla ${ }^{145}$.

Uno de los puntos planteados por Honigswald, en sus disciplinas, fue la fenomenología de Edmund Husserl. A pesar de su distancia teórica de esta concepción filosófica, el profesor de Filosofia e Historia de Breslau se sorprendió al descubrir que Edith Stein tenía la intención de ir a Gotinga para estudiar con el autor de Investigaciones lógicas. En el mismo semestre, Honigswald va a Gotinga para asistir a clases en el curso de Psicología, cuando se enfrenta a la fenomenología Husserliana:

Con toda la admiración que sentía por la sutileza de Honigswald, no podía pensar que se atreviera a compararse con Husserl. Ya estaba convencido de que Husserl era el filósofo de nuestro tiempo. Desde entonces, cuando el seminario en Honigswald hablaba de fenomenología, me utilizaron como "experta"146.

Edith Stein eligió, como tema que más le interesó durante los cuatro semestres que estudió en Breslau, la disciplina Introducción a la psicología, impartida por Willian Stern, quien también era psicólogo y filósofo, así como de origen judío y estudiante de Hermann Ebbinghaus. Esto presentó la escuela de pensamiento de Wurzburg, fundada por Oswald Kulpe, cuyo proyecto consistía en preguntar cómo los contenidos son aprehendidos por la conciencia a través de introspecciones controladas; sin embargo, con un enfoque solo en el pensamiento, ignorando otros aspectos de la conciencia. Uno de los intereses de Willian Stern era el estudio de la psicología experimental y el desarrollo cognitivo de niños y jóvenes. Desarrolló pruebas de inteligencia y fue responsable de introducir el término "edad mental" en psicología, proponiendo el cálculo del coeficiente de inteligencia ${ }^{147}$.

Stern representaba un tipo específico de humanismo judío. En ese momento, tenía poco más de cuarenta años, de estatura media, pero parecía ser más bajo, ya que estaba ligeramente inclinado. Una barba marrón enmarcaba la palidez de

145 ODO MARQUARD, Sobre la inevitabilidad de las ciencias del espíritu. In: O. Marquard, Apología de lo contingente. (Valencia: El Magnànim,2006), 538.

146 STEIN, Vida de uma família judia,600.

147 STEIN, Vida de uma família judia,306. 
su rostro, su mirada era inteligente y buena, la expresión de su rostro y el sonido de su voz expresaban una gran suavidad y una inmensa amabilidad. [...] Siempre dijo que en su corazón era filósofo (por eso siempre luchó contra la separación entre las cátedras de filosofía y psicología) y que su gran trabajo filosófico Persona y cosa era, en su opinión, lo más importante. de todos los demás. [...] Su trabajo sobre el lenguaje del niño y sobre la psicología de la primera infancia se basó en observaciones precisas de sus propios hijos y en el diario cuidadosamente guardado cada día por su esposa, quien, inteligente y amorosa, era el tu colaborador más leal ${ }^{148}$.

La descripción de Edith Stein de Stern, se basó en su contacto con el profesor no solo en la disciplina de Psicología, sino también en el grupo dedicado a los estudios pedagógicos, formado por estudiantes que tenían una profunda participación social en la formación de educadores. En ese momento, Stern desarrolló trabajos en el campo de la psicología experimental, en particular pruebas de medición de inteligencia. Este grupo incluía: Erna, la hermana de Stein, Hans Biberstein, Rose Guttmann, Lilli Platau y el médico y filósofo Georg Moskiewicz ${ }^{149}$. El último fue uno de los miembros que se presentó de manera prominente, y que estaba tratando de obtener Habilitación en el campo de la psicología, bajo la guía de Hermann Ebbinghaus, profesor de psicología experimental en Breslau. Después de la muerte de su consejero Moskiewicz, Edith Stein notó que estaba en un dilema, porque, aunque necesitaba la calificación para ejercer el cargo de profesor, no estaba de acuerdo con el método de psicología experimental utilizado por Stern. Stein consideró hacer un doctorado bajo la guía de William Stern, cuyos estudios se basaron en la observación cuidadosa de sus hijos, buscando entender cómo los contenidos son aprehendidos por la conciencia a través de introspecciones controladas, pero criticó el enfoque dado solo al pensamiento, ignorando otros aspectos de conciencia. Y esta fue la principal crítica hecha por Stein con respecto a la psicología:

En ese momento, él(Stern) estaba ocupado con métodos para medir la inteligencia. Así preparó su método para evaluar la aptitud profesional, que más tarde le valió el reconocimiento en Hamburgo. Tuvimos restricciones serias en todos estos temas, así como en su principio general de "solo medir". Su colega malicioso Honigswald habló un día sobre el tema de crear "psicólogos de posgrado": "El psicólogo de posgrado se convertirá en la persona más poderosa del estado". Él decidirá el trabajo de cada uno y, si está particularmente bien dispuesto a favor de alguien, lo 
designará para ser un psicólogo de posgrado ${ }^{150}$.

Stein dice que, a pedido de Stern, visitó a Otto Lipmann, un psicólogo de origen judío y pionero en el asesoramiento vocacional para jóvenes en el Instituto KleinGlienicke de Psicología Aplicada en Berlín, para mostrarle su trabajo El desarrollo del pensamiento de los niños. Después de la visita, Stein definitivamente decidió que ya no continuaría el proceso de doctorado en psicología:

Todos mis estudios en psicología me llevaron a la convicción de que esta ciencia todavía estaba en su infancia, ya que carecía de los fundamentos necesarios para ideas básicas y claras, y que no podía elaborar estos supuestos. Por otro lado, lo que ya sabía sobre fenomenología me entusiasmó, porque consistía fundamental y esencialmente en un trabajo de aclaración y que, desde el principio, había forjado los instrumentos intelectuales que necesitaba ${ }^{151}$.

Durante el semestre de 1913, el profesor Willian Stern sugirió que sus alumnos presentaran un seminario cuyo tema sería la escuela de Würzburg, de la que formaban parte. Al estudiar sobre estos autores, Stein tiene su primer contacto con el trabajo Investigaciones Lógicas de Husserl, que fue citado constantemente por los autores de la escuela. A través de la guía de Georg Moskiewicz, que estudiaba las disciplinas enseñadas por Edmund Husserl y presentó las Investigaciones Lógicas a Edith Stein, se le advirtió que "en Gottingen, no hay nada más que filosofar día y noche, en las comidas y en la calle. En todas partes. Solo hablamos de fenómenos " 152 .

Para comprender la influencia que el pensamiento de la escuela fenomenológica tuvo en el trabajo de Edith Stein, será esclarecedor demostrar cómo el pensamiento de esta escuela filosófica estuvo presente en Europa, más necesariamente en la escuela de Würzburg. El enfoque de la psicología a la escuela fenomenológica se puede ver a través del asesor de calificación docente de Edmund Husserl en Halle, el filósofo Carl Stumpf. Su influencia en el trabajo de Husserl se puede ver en el hecho de que su trabajo principal: Investigaciones Lógicas se dedicó a Stumpf (Spiegelberg, 1982, p.64). Además de Husserl, el filósofo Franz Brentano y los psicólogos Wolfgang Köhler y Kurt Koffka fueron instruidos por el profesor de Halle.

150 STEIN, Vida de uma família judia e outros,306.

151 EDITH STEIN, Escritos Antropológicos y Pedagógicos: Magisterio de vida cristiana, 1926-1933. (Madrid: El Carmen,2002),331.

152 STEIN, Escritos Antropológicos y Pedagógicos, 334. 
Herbert Spiegelberg señala que, a diferencia de Husserl, su supervisor, el psicólogo y filósofo Carl Stumpf tenía una mayor apertura a la psicología y otras áreas del conocimiento; sin embargo, a pesar de las diferencias metodológicas, ambos buscaron analizar el método fenomenológico a través de la descripción detallada de la experiencia inmediata de los fenómenos y, a partir de esta descripción, comprender la estructura de los fenómenos ${ }^{153}$ Eduardo González di Pierro señala que Edith Stein también forma parte del mismo conjunto de críticas al psicologismo de su maestro en Gotinga, Edmund Husserl ${ }^{154}$.

\section{CÍRCULO FENOMENOLÓGICO DE GOTTINGEN}

En abril de 1913, Edith Stein decidió irse a Gotinga. Este cambio se produjo debido a varios factores, entre ellos el contacto con Investigaciones Lógicas, presentado por Willian Stern y Richard Honigswald. Está fascinada por el trabajo de Husserl, convencida de que debería tomar clases con el propio autor. Otros factores responsables de esa decisión fueron: leer el texto Los fundamentos epistemológicos del positivismo de un joven estudiante de Husserl, Conrad-Martius, quien inspiró a Stein; Además, Richard Courant, primo de Edith Stein, fue nombrado profesor de matemáticas en Gotinga y se ofreció a la Sra. Augusta Courant Stein para recibir a sus hijas Edith y Erna para completar su educación universitaria allí ${ }^{155}$.

A la edad de veintiún años, Edith Stein llega a la ciudad de Gotinga y en su diario, La vida de una familia judía, trata de describirla como opuesta a Breslau, concibiéndola como una verdadera ciudad universitaria, con aproximadamente treinta mil habitantes, siendo la mayoría de ellos inmigrantes, por lo que era una ciudad plural. Una ciudad donde el pasado también fue un signo sorprendente, especialmente en la arquitectura de edificios y signos

Los carteles conmemorativos que se encontraban en casi todas las casas antiguas me llamaron la atención: indicaban a las personas famosas que habían vivido en ellas. El pasado recordado a cada paso: los hermanos Grimm, los físicos Johann Carl Friedrich Gauß y Wilhelm Eduard Weber y otros que pertenecían a 'los siete de Gottingen', todos ellos, habían vivido y hecho algo aquí, y estuvieron presentes

153 HEBERT SPIELGELBERG, Phenomenology in psychology and psychiatry: a historical introduction. (Evanston: Northwestern University Press, 1972),33.

154 EDUARDO GONZALEZ PIERRO, La fenomenología de Edith Stein como refutación del "realismo fenomenológico" del "Círculo de Gotinga". in Stein y su interpretación del idealismo trascendental husserliano. (Ciudad de México,2004), 159.

155 STEIN, Vida de uma família judia,342. 
para el recuerdo en futuras generaciones ${ }^{156}$.

Para Stein, Gotinga fue una experiencia de buceo en el pasado, en lugares donde vivían grandes hombres como los hermanos Jakob y Wilhelm Grimm, que formaban parte del "grupo de siete en Gotinga" junto con los historiadores Friedrich Christoph Dahlmann y Georg Gottfried Gervinus como Otto Von Bismarck, que había sido estudiante en la universidad. Fue en esta inmersión que Edith Stein pasó durante las vacaciones antes del comienzo del período escolar en Gottingen.

Siguiendo el consejo de Georg Moskiewicz, quien la había recibido en Breslau, dijo que "cuando llegó a Gotinga, lo primero que tuvo que hacer fue buscar a Adolph Reinach y él se encargaría del resto" ${ }^{157}$. Adolph Reinach trabajó como asistente de Edmund Husserl, a cargo del curso de iniciación en fenomenología, proveniente de Múnich, y también fue responsable de su primera entrevista en la universidad.

Escuché que alguien caminaba, la puerta se abrió y me encontré frente a Reinach. Era de mediana estatura y tenía hombros cuadrados, sin ser robusto. A través de las lentes de las gafas de pince-nez (sin tallo), los ojos marrones miraron al interlocutor con una mirada inteligente y extremadamente acogedora. Me saludó amablemente, me invitó a sentarme en el sillón más cercano al escritorio. [...] 'El señor Moskiewicz me escribe sobre ti. Ya estudiaste Fenomenología, ¿verdad? Le di algunas explicaciones rápidas. "Estaba dispuesto a aceptarme de inmediato en su" trabajo práctico para estudiantes avanzados ", pero todavía no podía decirme exactamente el día y la hora, ya que primero necesitaba combinarlos con sus estudiantes. Prometió presentarme a Husserl ${ }^{158}$.

Adolf Reinach buscó la fenomenología de Husserl a partir de las críticas hechas al psicologismo, generalmente centradas en la figura de su ex profesor Theodor Lipps, así como en los otros miembros del primer círculo de fenomenología realista. El proyecto de Lipps, según lo definido por Macintyre, fue el desarrollo de la lógica a partir de la explicación de la actividad mental proporcionada por la psicología empírica. Esta perspectiva, como señalamos anteriormente, fue contrastada por la concepción de Husserl, para quien la sumisión de la lógica a la psicología era un error que necesitaba ser combatido, siendo esta crítica una parte

156 STEIN, Escritos Antropológicos y Pedagógicos, 347.

157 Ibíd, 352.

158 STEIN, Vida de uma família judia, 354. 
central del trabajo Investigaciones Lógicas, que, para esta primera generación del círculo fenomenológico de Gottingen, fue "un trabajo que redefinió la filosofía para ellos"159.

Sin embargo, Edith Stein fue fuertemente influenciada por la crítica del psicologismo y por el proyecto filosófico propuesto por Husserl, así como por el trabajo de otros miembros de la línea fenomenológica realista, como Adolph Reinach, Hedwig Conrad-Martius y Hans Lipps, como señaló Eduardo González di Pierro ${ }^{160}$, Edith Stein recorrerá su propio camino dentro del Círculo de Gottingen ${ }^{161}$.

En diferentes lugares y publicaciones, indicamos nuestras objeciones al colocar a Edith Stein en la llamada línea fenomenológica realista que caracterizó a la escuela de Munich-Gotinga. Ciertamente, al principio, nuestro filósofo fue fuertemente influenciado por Reinach y, especialmente, por su amigo y madrina, Hedwig Conrad-Martius, y, en consecuencia, compartió ampliamente la perspectiva fenomenológica realista. Muy pronto, esta posición comenzó a ser modificada por la estrecha colaboración de Stein con el propio Husserl como asistente. Ella sola lo siguió a Friburgo y gradualmente adquirió una mayor comprensión de lo que podríamos llamar el esquema general de la filosofía fenomenológica de Husserl. Se distanció de los puestos ocupados, además de Reinach, Hedwig-ConradMartius, Roman Ingarden, Jean Héring y, aunque quizás en menor medida, de otros miembros del Círculo de Göttingen .

Edith Stein estaba encantada con su entrevista con Adolf Reinach, a quien describe como "una persona con un corazón puro"162, de modo que, después de esta reunión, Reinach fue responsable de iniciar ella en su clase de introducción a la fenomenología todos los lunes de 18:00 a 20:00. Lamentablemente, no pudo participar, ya que era el momento del Seminario de Historia impartido por Max Lehmann ${ }^{163}$.

En la presentación del seminario, los estudiantes se sentaron en una mesa con forma de herradura, frente al profesor Max Lehmann, para responder sus preguntas. Esta organización del aula tenía la intención, según el propio Lehmann,

159 MACINTYRE, Edith Stein: A Philosophica Prologue 1913-1922, 17.

160 EDUARDO GONZALEZ PIERRO, The Influence of Adolf Reinach on Edith Stein's Concept of the State: Similarities and Differences. (Ciudad de México,2016),94.

161 PIERRO, The Influence of Adolf Reinach on Edith Stein's Concept, 94.

162 STEIN, Escritos Antropológicos y Pedagógicos, 354.

163 Ibíd, 354. 
de acercarse e interactuar entre los estudiantes, además de que el maestro también tenía una visión muy débil y no reconocía a los estudiantes desde la distancia. El tema sugerido a Edith Stein y su compañero fue la Asamblea para la Constitución del Imperio Alemán, $1849{ }^{164}$.

Como resultado del trabajo sobre la Constitución de 1849, Max Lehmann sugirió que el trabajo presentado en el seminario debería ser el tema de investigación que Stein debería someter a evaluación para el examen del Estado, bajo su guía en Breslau, sin embargo, la sugerencia del proyecto es rechazada por Edith Stein, quien ya había decidido que tenía la intención de ser estudiante de Husserl y pertenecer al Círculo de Gottingen.

Ahora, lo que tenía que hacer de inmediato era organizar mis relaciones con el profesor Stern. Le envié un informe sobre el progreso del semestre: no hice nada en relación con mi trabajo en psicología; por el contrario, estaba completamente involucrado en fenomenología. Ahora, mi deseo ardiente era seguir trabajando con Husserl. Recibí una respuesta muy favorable. Si este fuera realmente mi deseo, él, a su vez, tenía que darme solo un consejo: hacer su doctorado con Husserl. Tampoco encontré resistencia en mis parientes ${ }^{165}$.

A pesar de su interés en la disciplina de la Historia Moderna en Alemania, Edith Stein decidió dejar de participar en el Seminario de Lehmann debido al conflicto de horarios con el grupo de Introducción a la Fenomenología impartido por Adolf Reinach, quien en ese semestre de 1913 estudió el libro O Formalismo en la ética y la ética material de los valores Max Scheler es otro filósofo alemán (de origen judío) que influiría en gran medida en el trabajo de Edith Stein, principalmente desde el concepto de "persona humana" y la empatía, ambos propios de la tradición filosófica católica, que, es notable, Edith Stein poco Sabía hasta entonces.

Para mí, como para muchos otros, la influencia (de Scheler) en esos años fue muy grande, más allá del ámbito filosófico [...]. En ese momento tenía muchas ideas católicas y sabía cómo difundirlas utilizando su inteligencia y su poderosa elocuencia. Así fue como entré en contacto con un mundo que hasta entonces era totalmente desconocido para mí. Esto todavía no me llevó a la fe, pero abrió un campo de "fenómenos" al que ya no podía estar ciego. No fue por nada que

164 STEIN, Escritos Antropológicos y Pedagógicos, 371.

165 Ibíd, 374. 
recomendé considerar todo con ojos libres de prejuicios ${ }^{166}$.

En 1899, Max Scheler se había convertido al catolicismo, pero después de un período de desapego regresó a la iglesia en 1914, en el mismo período en el que trabaja en Gotinga, donde impartió seminarios, en los que Edith Stein fue una de las estudiantes. Stein se sentía más cercano a Husserl, por su "gran honestidad intelectual" ${ }^{167}$, pero también admiraba los seminarios ofrecidos por Scheler, a quien describe como "brillante y seductora, pura expresión del fenómeno del genio" 168 .

En la primera parte (del problema de la empatía), respaldada por algunas notas tomadas de los cursos de Husserl, examiné el acto de "empatía" como un modo particular de conocimiento. Sobre esta base, abordé un tema que siempre me interesó especialmente y que luego me ocupó en todos los trabajos posteriores: la constitución de la persona humana. En relación con mi primer trabajo, esta investigación fue necesaria para mostrar cómo la comprensión de las asociaciones mentales difiere de la simple percepción de los estados psíquicos. Con respecto a estos temas, los cursos y escritos de Max Scheler, así como los trabajos de Wilhelm Dilthey, fueron de gran importancia para mí ${ }^{169}$.

Edith Stein finalmente conoce a Edmund Husserl y lo describe como "un maestro distinguido típico de estatura promedio y apariencia digna" ${ }^{170}$. Había leído el segundo volumen del trabajo Investigaciones lógicas, que impresionó a Husserl y pronto admitió a Stein en su escuela de Filosofía con las siguientes palabras: Edith Stein, eres una heroína por haber leído mi trabajo ${ }^{171}$.

El contacto de Edith Stein con la fenomenología ocurrió de una manera muy intensa, y ella fue profundamente influenciada por el método fenomenológico de Husserl. La filósofa utilizó el método fenomenológico en sus investigaciones, ya que vio la fenomenología como una posibilidad exhaustiva, estimulante y rigurosa de investigar fenómenos, lo que la ayudó en su búsqueda de la verdad. La trayectoria de Edith Stein en fenomenología fue muy peculiar, ya que no se limitó a los análisis de Husserl, sino que también desarrolló su propia concepción de la fenomenología. Stein tuvo una intensa trayectoria de vida, dedicada principalmente a cuestiones

166 STEIN, Escritos Filosóficos Etapa fenomenológica, 306.

167 STEIN, Escritos Antropológicos y Pedagógicos, 356.

168 Ibíd, 305.

169 STEIN, Vida de uma família judia, 466.

170 STEIN, Escritos Antropológicos y Pedagógicos, 356.

171 Ibíd, 355. 
relacionadas con el ser humano y la búsqueda de la verdad. Es posible afirmar que su vida y su trabajo están confundidos, construyendo este gran pensador de nuestro tiempo.

En su curso sobre la naturaleza y el espíritu, Husserl había dicho que un mundo externo objetivo solo puede ser experimentado intersubjetivamente, por una pluralidad de individuos conscientes, que están situados en un intercambio cognitivo. De acuerdo con esto, se supone la experiencia de otros individuos. Para esta experiencia peculiar, Husserl, siguiendo los trabajos de Theodor Lipps, llamado "empatía", sin embargo, [...] era una brecha que necesitaba ser explicada: quería investigar qué era la "empatía". Este hecho no desagradó al maestro; sin embargo, aún tenía que enfrentar un amargo desafio: le pidió a (Husserl) que realizara mi trabajo en confrontación con Theodor Lipps ${ }^{172}$.

Edmund Husserl explica que la fuente del proyecto fenomenológico de una filosofía universal, incluso en sus diferentes enfoques o en las diferentes formas de reducción, es siempre y constantemente la revelación del propio ser. Por lo tanto, el objetivo de epoché es la reducción al yo absoluto como centro para comprender cualquier constitución ${ }^{173}$. Según Ângela Alles Belo ${ }^{174}$, una parte esencial para comprender "la nueva forma" propuesta por Husserl en el libro Crisis de las ciencias europeas es el cambio epistemológico, en el que el ser humano se convierte en el punto central de la descripción. y análisis de las universalidades de la experiencia humana. Tal proceso solo es posible, para Edmund Husserl, a partir de la percepción de que las dimensiones del ser humano solo pueden entenderse mientras esté compuesto de cuerpo, psique y espíritu: "La espiritualidad humana ciertamente se basa en la fisis humana, cada una de las vidas individuales del alma humana se basa en la corporeidad y, por lo tanto, cada comunidad también se basa en los cuerpos de los individuos humanos que son miembros de esa comunidad" 175 .

En este proceso, para la comprensión y revelación del ser humano y su relación con fenómenos singulares y universales, las ciencias humanas serían la forma de entender eso, incluso en períodos de inestabilidad, como la Segunda Guerra

172 STEIN, Escritos Antropológicos y Pedagógicos, 356.

173 EDMUND HUSSERL, La crisi delle scienze europee e la fenomenologia trascendentale. (Saggiatore Milano, 1960),213.

174 ANGELA ALES BELLO, A Questão do Sujeito Humano. In: Anais IV Seminário Internacional de Pesquisa e Estudos Qualitativos. (São Paulo, 2010),12.

175 HUSSERL, La crisi delle scienze europee, 13. 
Mundial, a través de la epoché, podrían crear lazos de amistad y comprender que, incluso si el sujeto moderno es plural, tiene características universales que se comparten.

Esta tradición de empatía, que no puede reducirse a una pura tradición hermenéutica, estuvo presente en la escuela fenomenológica de Gotinga a través, por ejemplo, del trabajo sobre la percepción de Theodor Lipps del campo de la estética, siendo las influencias de Wilhelm Wundt y Edmund Husserl confesó el propio autor ${ }^{176}$, así como su colega Edith Stein, para quien el tema central y el ser humano, y la relación entre fenomenología y antropología filosófica.

Con el fin de comprender a la persona humana en su pluralidad y de él para crear lazos de empatía y afecto, Edith Stein señala que las ciencias humanas, especialmente la historia, podrían ser el camino para la restauración y la creación de lazos de identidad y orientación, porque por las experiencias, los hombres se percibirían como cercanos entre sí. Esto significa que tendrían la capacidad de compensar la especificidad de las ciencias biológicas, como presentamos en la crítica del psicologismo.

Encontramos la concepción de que la psicología estaría en la base de la historia en los manuales del método histórico. [...] Definitivamente no queremos argumentar que las cogniciones psicológicas no pueden ser útiles para el historiador. Estas concepciones pueden ayudarlo a comprender lo que es externo a su campo, pero no pueden ofrecerle sus propios objetos. Puedo explicar psicológicamente las acciones de una persona en la historia, pero cada vez que hago eso, procedo como estudiante de ciencias naturales y no como historiador. [...] Lo que hay que "entender" es cómo los fenómenos naturales, cuando se presentan, motivan las acciones de las personas y cómo están "motivados", pero estos fenómenos tienen un significado histórico. En este caso, tales fenómenos ya no se conciben como hechos naturales que deben ser explicados por las leyes naturales. Si hubiera "explicado" todos los componentes biológicos de las personas que vivieron en el pasado, habría hecho un buen trabajo en las ciencias naturales, pero habría destruido por completo el espíritu cultural y no habría adquirido ni un grano de conocimiento histórico. Si los historiadores consideran que su trabajo es asegurar y explicar los hechos psicológicos del pasado, entonces ya no hay una ciencia de la historia ${ }^{177}$.

176 THEODOR LIPPS, Die Aufgabe der Psychologie. in: E. Stein, Escritos Filosóficos Etapa fenomenológica: 1915-1920. (Madrid: El Carmen,1933),141.

177 EDITH STEIN, II problema dell'empatia, de E. in: E. Costantini \& E. Schulze-Costantini. (Roma: Studium,1998), 199. 
En la conferencia sobre la Estructura de la Persona Humana, Edith Stein describe cómo la Escuela de Ciencias Políticas de Baden, especialmente los filósofos Heinrich Rickert y su asesor Wilhelm Windelband, trataron de definir las ciencias en dos grupos principales: el nomotético (buscar leyes universales) y el ideográfico (que describe estructuras individuales). Sin embargo, a diferencia de Rickert y Windelband, las ciencias humanas no podrían reducirse solo a las individuales, porque su objeto, la persona humana, se compone de pluralidad; por lo tanto, la concepción de Edith Stein de la persona humana es necesariamente moderna.

Esta división se superpone con lo que otros prefieren entre las ciencias naturales y las ciencias humanistas. Las ciencias naturales pueden considerarse nomotéticas porque, incluso cuando procedemos de manera descriptiva, buscamos una ley universal de formación y estudiamos al individuo siempre solo como un ejemplo, y nunca en su individualidad. Por otro lado, no es posible equiparar las ciencias idiográficas con las del espíritu. Hay ciencias humanistas que se ocupan de algo único: la historia busca investigar y exponer la marcha de la humanidad a lo largo del tiempo, como ha sucedido de una manera única e irrepetible, en individuos humanos y pueblos concretos ${ }^{178}$.

Edith Stein da fe de que la historia, especialmente la Filosofia de la Historia, tendría características universalistas, al igual que las propias herramientas del historiador tendrían una apertura a la dimensión universal, ya que continuarían existiendo separadas de la materialidad del sujeto, como, por ejemplo, los objetos de estudios, como cartas, un testamento o un texto literario, que pueden sobrevivir en relación con quien los escribe y al mismo tiempo pueden afectar a otros hombres. Por lo tanto, la tarea preliminar del historiador, para Stein, sería recopilar estas fuentes; sin embargo, afirma que la tarea principal sería comprender estos testimonios y de ellos "penetrar en la individualidad a través del lenguaje de estos signos" ${ }^{179}$. Por lo tanto, parece que la misión del historiador sería el paso de esta individualidad a la universalidad a través de la escritura, teniendo así la posibilidad de crear lazos de afecto:

Luego viene la misión de poner a disposición de otros la individualidad que ha sido capturada. Tal fin no puede lograrse dando a la individualidad una denominación universal o enumerando muchas de sus características (a su vez, universalmente comprensibles), ni viéndola como la intersección de diferentes tipos. Todos estos son solo herramientas que se pueden utilizar. Pero, lo que es importante para

178 EDITH STEIN, Estrutura da pessoa Humana. (Madrid: El Carmen. 1933), 585.

179 STEIN, Estrutura da pessoa Humana, 588. 
permitir que alguien capte una individualidad cuando no se puede proporcionar un encuentro vivo es señalar el camino por el cual se alcanzó la meta. Para que el acto de comprensión sea cooperativo, se deben informar los rasgos especialmente elocuentes y, sobre todo, siempre que sea posible, ofrecer expresiones originales de la persona en cuestión ${ }^{180}$.

Eduardo González di Pierro señala que el análisis del objeto histórico para Edith Stein debería comenzar desde las experiencias. Esta concepción es contraria a la interpretación del sociólogo George Simmel, desde el cual los eventos reales son continuos, pero las descripciones sobre ellos, como los eventos históricos, buscan crear una cierta orientación, a través de una narrativa que presenta estos eventos como incrustados en una idea unitario. Stein le escribe a Simmel: "Descomponiendo esta unidad aún más, finalmente llegaremos a átomos que ya no tienen importancia histórica y sobre los cuales, por lo tanto, la historia ya no se puede construir" ${ }^{\prime 181}$.

Odo Marquard señala que, cuando elaboramos un trabajo científico en el área de las ciencias humanas, el pluralismo moderno podría contribuir porque, cuando el científico social busca pensar en un problema, se acerca a otros científicos que piensan en este problema en común o parten de los mismos objetos, ser capaz de proponer diferentes análisis que ayudarían a comprender el fenómeno en su conjunto ${ }^{182}$. De manera similar, podemos analizar el pensamiento steiniano, que, si se compara con el de Simmel, revela que, a pesar de los múltiples aspectos de la escritura historiográfica, contribuyen a comprender la realidad del fenómeno estudiado, nadie puede representarlo de manera exhaustiva, porque:

[...] ciertamente el historiador de la política, la economía y el arte da noticias muy diferentes del mismo período histórico (...). Sin embargo, esto solo significa que el contenido del significado del evento original era algo multiforme, no que cada persona le asignara un significado diferente ${ }^{183}$.

Con el advenimiento de la Primera Guerra Mundial, muchos de los miembros del Círculo de Gottingen fueron llamados a luchar en el frente, lo que, según Hans Rainer Sepp, interrumpió el proyecto filosófico del Círculo de Gottingen, que

180 Ibíd, 589.

181 PIERRO, La fenomenología de Edith Stein como refutación del "realismo fenomenológico" del "Círculo de Gotinga”, en Stein y su interpretación del idealismo trascendental husserliano. (Ciudad de Mexico, 2004), 157.

182 MARQUARD, Sobre la inevitabilidad de las ciencias del espíritu, 153.

183 HANS SEPP RAINER, La Postura de Edith Stein dentro del movimento fenomenológico. (Prahal Ed. Akademie ved Ceské,1998),283. 
también debe como factor la transferencia de Husserl a Universidad de Friburgo en $1916{ }^{184}$. Sin embargo, fue el evento de la Primera Guerra Mundial el que más afectó al grupo de filósofos fenomenológicamente orientados ${ }^{185}$, como Dietrich von Hildebrand, que se desempeñó como enfermero en Munich, Adolf Reinhard, quien se unió al ejército y recibió el Cruz de hierro muriendo en la batalla del Somme, en Flandes, el 16 de noviembre de 1917.

Edith Stein se sintió obligada a actuar en la Primera Guerra Mundial, ya que se consideraba una "verdadera ciudadana alemana" ${ }^{186}$. Poco después de los exámenes de griego e historia en Breslau, Stein contactó a la Cruz Roja de la ciudad para alistarse, pero respondió que en Alemania no había demanda, ya que el personal de enfermería ya estaba completo. En Austria había una gran necesidad, por lo que la llamaron al hospital de Mährisch Weißkirchen a principios de abril y, según Stein, estaba en la región de Moravia ${ }^{187}$. Los soldados con cólera, tifus y otras fiebres o infecciones agudas fueron enviados allí durante la Primera Guerra Mundial, lo que significa que Mährisch Weißkirchen era un lugar donde la actividad principal era atender a los pacientes en cuarentena.

El 7 de abril de 1915, Edith Stein viajaba en tren desde Göttingen a Mährisch Weißkirchen, con la insignia de la Cruz Roja, llegando al mediodía. La ciudad que describe como pequeña es agradable, ya que tiene como edificio central la antigua academia militar que se había transformado en un hospital, bajo la dirección de Margareth, una vieja enfermera que trabajaba en la ciudad. Edith Stein era parte del equipo de ciento cincuenta enfermeras que trabajaban en el hospital, y fue asignada a trabajar en la sala de tifus.

El personal del hospital estaba formado por médicos, enfermeras y enfermeras en su mayoría; por miembros de todas las diferentes naciones que pertenecían a la monarquía austrohúngara, es decir, alemanes, checos, eslovenos, polacos, húngaros, romanos, italianos, así como turcos, rusos y gitanos, y para la operación del hospital todos siguieron un libro estándar con preguntas y respuestas, que fue escrito en nueve idiomas ${ }^{188}$. Creemos que este contacto condujo, a través de experiencias, es decir, de vivir con otras personas, especialmente de otras nacionalidades, a analizar el fenómeno de la empatía. Como podemos ver en la

184 RAINER, La Postura de Edith Stein, 710.

185 JOHN TERRICABRAS, Dicionário de Filosofia. (São Paulo Ed: Loyola, 1994),1230.

186 STEIN, Escritos Antropológicos y Pedagógicos, 103.

187 EDITH STEIN, Psicologia e scienze dello spirito. Contributi per una fondazione filosofica. (Roma: El Carmen, 1996),231.

188 STEIN, Escritos Antropológicos y Pedagógicos, 427. 
descripción de Edith Stein de uno de sus pacientes:

Era un joven comerciante italiano, de Trieste. Solo lo llamaron por su nombre, Mario; No recuerdo tu apellido. La enfermedad lo atacó violentamente. Su boca estaba llena de una secreción mezclada con sangre. La enfermera Loni me pidió que se limpiara la boca con un paño cada vez que lo pasaba. Me agradeció con la mirada por ese gesto caritativo. No pude decir nada porque había perdido la voz por completo. En cada visita médica, fue examinado a fondo. Médicos y enfermeras hablaron de él, a su lado, como si no entendiera nada. Pero pude ver en sus grandes y brillantes ojos que estaba perfectamente consciente, prestando atención a cada palabra que decía ${ }^{189}$.

Edith Stein dedica un subcapítulo completo de su biografía, La vida de una familia judía, para describir cómo se relacionaba con los diferentes pacientes, y este contacto con los diferentes pueblos, lo que la motivó a seguir trabajando, como sus compatriotas alemanes, que ella describió. tan exigente y crítico, como los eslovenos, a quienes Stein describe como humildes, del mismo modo que se describe el coraje de los soldados húngaros y los soldados checos. Cuando no estaba cuidando a los diferentes pacientes, Edith Stein buscó continuar su educación leyendo los dos libros que había elegido llevar al hospital, que eran Ideas para la fenomenología pura y para una filosofía fenomenológica: Introducción general a la fenomenología de Husserl y la Odisea de Homero.

Después de cinco meses de arduo trabajo y dedicación a sus pacientes, Edith Stein se da cuenta de que sus nervios estaban llegando al límite, necesitaban descansar y, al mismo tiempo, sintió la necesidad de volver a los estudios a partir del conjunto de experiencias que había aprendido. Regresando así a su hogar en Breslau en septiembre de ese año. El hospital Mährisch Weißkirchen fue cerrado un mes después. Debido a su dedicación y dedicación a trabajar como enfermera, Stein recibió la medalla de la Cruz Roja por su valor.

\section{GONSIDERAGIONES FINALES}

Entendemos que a lo largo de los latros de la escritura biográfica desarrollada por Edith Stein en sus escritos en la obra Vida de una familia judía y otros escritos autobiográficos, podemos ver que su primer interés por comprender a la persona humana se desarrolló en el área reciente de la psicología, curso que tomó en su

189 EDITH STEIN,"Live in a Jewish Family - her unfished autobiographical account" in The Collectec Works of Edith Stein. (Washington: Publications, 1986),416. 
ciudad natal Breslau a través del psicoanalista William Stern con quien aprendió cómo se desarrolla la investigación científica y académica, como los conceptos de "mónadas" desarrollados por Stern, pero cómo esta ciencia aún presentaba sus fundamentos y presentaba un carácter conductista llevó a cierto desencanto a Edith Stein sobre psicología.

Sin embargo, es através de las clases de William Stern que Edith Stein conocerá el trabajo de Edmund Husserl Investigaciones lógicas, es a través de la lectura de este trabajo que llevará al intelectual a desarrollar una filosofía a través de la corriente fenomenológica de Husserl, y buscará desarrollarse en su tesis doctoral sobre El Problema de la Empatía, una reanudación del diálogo entre la fenomenología de Husserl y la psicología que aprendió de Stern, que le permitió comprender que el fenómeno empático no puede reducirse simplemente a un fenómeno psíquico como señala Theodor Lipps en el trabajo La tarea de la psicologia,sino a un fenómeno de experiencias.

Del breve análisis de los textos en los que Stein presenta su concepción y comprensión de la fenomenología, se descubrió, primero, la aprensión singular que este importante alumna y discípula de Edmund Husserl tenía del método fenomenológico. De acuerdo con Husserl, Stein presenta la Fenomenología como un método que busca regresar a las cosas mismas y alcanzar la esencia de los fenómenos. Para esto, la fenomenología utiliza el método intuitivo, a través del cual es posible aprehender las esencias, que se muestran de inmediato. En segundo lugar, Stein también destacó la reanudación de la idea de la verdad absoluta y la objetividad de la conciencia, promovida por Husserl con la publicación de sus Investigaciones Lógicas, que rompió con las filosofias relativistas vigentes en ese momento y rescató el rigor de la investigación filosófica: "no pueden satisfacer los significados que cobran vida, cuando lo hacen, desde intuiciones remotas, confusas e inapropiadas" ${ }^{190}$.La filósofa estuvo de acuerdo con el maestro, y como destacamos en su biografía, esa filosofía, y especialmente las ciencias, carecía de una base radical y eso elevaba la filosofía a una "ciencia en sentido estricto" ${ }^{191}$.

Concluimos, por lo tanto, que Stein presentó una concepción original de la fenomenología husserliana, incluso en un momento en que seguía diferentes

190 EDITH STEIN, Los tipos de psicología y su significado para la pedagogía. (Madrid: El Carmen,2002),311. 191 EDMUND HUSSERL, Ideias para uma fenomenologia pura e para uma filosofia fenomenológica. (Aparecida: Ideias \& Letras, 2006),3. 
caminos a través de la crítica de Husserl. La filósofa permaneció fiel a la filosofía de su maestro de muchas maneras, al mismo tiempo que desarrolló sus propias nociones, buscando complementar el proyecto husserliano, sin embargo, caracterizarlo erróneamente. En este sentido, la autonomía y la originalidad presentadas por Stein revelan su notable comprensión de la fenomenología, en vista de los innumerables conceptos erróneos que existían en ese momento. 